\title{
Residual Impact of Integrated Nutrient Management on Yield Attributes, Yields, Nutrient Uptake and Economics in Wheat (Triticum aestivum)
}

\author{
Narendra Singh ${ }^{1 *}$ and H. S. Kushwaha ${ }^{2}$ \\ ${ }^{1}$ Department of Agronomy, Banda University of Agriculture and Technology, \\ Banda, UP 210001), India \\ ${ }^{2}$ Mahatma Gandhi Chitrakoot Gramoday Vishwavidhyalay, Satna, MP, India \\ *Corresponding author
}

\begin{abstract}
A B S T R A C T
Keywords

Residual impact, Yield, Nutrient uptake, Economics, Wheat

Article Info

Accepted:

10 August 2020

Available Online:

10 September 2020

A field experiment was conducted at Deendayal Research Institute Krishi Vigyan Kendra Chitrakoot UPto assess the residual impact of Integrated Nutrient Management and direct effect of inorganic fertilization on Wheat (Triticum aestivum) for yield attributes, yields, nutrient uptake and economics during Rabi season of 2008-09 and 2009-10. Total 18 treatment included residual effect of 3 different doses of inorganic fertilizers and 3 different manures (FYM, Vermi-compost and Cow Pat Pit) and direct 3 doses of inorganic fertilizers to wheat crop were tested. The yield attributing characters of wheat like Spike length $(\mathrm{cm})$, No. of spikes $\mathrm{m}^{-1}$, No. of spikes plant ${ }^{-1}$, No. of spikelet spike ${ }^{-1}$. No. of seed spike $^{-1}$, Seed weight $\mathrm{g}$ spike ${ }^{-1}$, Seed weight $\mathrm{g} 1000^{-1}$ had been found significantly higher with application of $100 \%$ NPK to wheat + residual effect of 50\% NPK and FYM @ 10 tha $^{-}$ ${ }^{1}$ during two years. Seed yield (4.94 and 4.95 tha $^{-1}$ ), straw yield (4.95 and 5.04 tha $^{-1}$ ) and harvest index (49.95 and $49.54 \%$ ) of wheat were also obtained highest with same treatment combination during 2008-09 and 2009-10. The NPK uptake by wheat crop was also found maximum (119.0 and $123.3 \mathrm{~kg} \mathrm{ha}^{-1} \mathrm{~N}, 20.3$ and $20.0 \mathrm{~kg} \mathrm{ha}^{-1} \mathrm{P}, 82.0$ and $83.6 \mathrm{~kg}$ $\mathrm{ha}^{-1} \mathrm{~K}$ ) with this treatment. Similarly, residual impact of 50\% NPK and FYM @ 10 tha $^{-}$ ${ }^{1}$ and 100 recommended dose of fertilizers to wheat also obtained significantly higher values of Gross return (Rs.54372 and $62083 \mathrm{ha}^{-1}$ ), Net return (Rs. 42479 and $48213 \mathrm{ha}^{-1}$ ) and $\mathrm{B}: \mathrm{C}$ ratio (4.57 and 4.48 ) during both years.
\end{abstract}

\section{Introduction}

Wheat (Triticum aestivum) occupies the prime position among the food crops in the world. In India, it is the second important food crop being next to rice and contributes to the total food grain production of the country to the extent of about $25 \%$. Wheat has played a vital role in stabilizing the food grain production in the country over the past few years. Wheat production has increased tremendously but is still far below the potential yield (11.2 tha $\left.{ }^{-1}\right)$ (Singh et al., 2010). Although, India is well placed in meeting its needs for food grains with major objective of food and nutritional security for its entire population has not been achieved. The demand for food grains is expected to rise not only as a function of 
population growth but also as more and more people cross the poverty line with economic and social development (Parewa et al., 2019).

On account of continuing world energy crisis and spiraling price of chemical fertilizer, the use of organic manure as a renewable source of plant nutrients is assuming importance. In this endeavor proper blend of organic manure and inorganic fertilizer is important not only for increasing yield but also for sustaining soil health (Kumar et al., 2013).Continuous use of chemical fertilizers has led to problem of soil degradation, which is proving detrimental to crop production (Das et al., 2015). Application of organic material along with inorganic fertilizers into the soils leads to increase in productivity of the cropping system enhance the use efficiency of fertilizer input and sustain $\mathrm{n}$ the soil health for longer period (Jat et al., 2015). Fertilizer is one of the costliest inputs of crop production, it is therefore, very important to find out the way of economic use of fertilizer (Dhakal et al., 2016).

Bundelkhand region of Uttar Pradesh has limited irrigation facilities with heavy soils. The scenario of agriculture in this region is suitable for integrated nutrient management which comprises with organic and inorganic fertilizers. Wheat is only important crop of Bundelkhand region in winter season under irrigated condition, which contributes major part of human diet and economy.

The increase in grain yield of wheat due to balanced fertilization is attributed to improvement in growth and yield attributes, which in turn resulted in higher translocation of photosynthates and nutrients, ultimately reflected into higher grain and straw yield (Chandel et al., 2014 and Singh et al., 2018). Hence, the present study was planned and carried out to explore residual impact of organic manures and inorganic fertilizers to succeeding crop of wheat under different level of fertilizer management on yields, nutrient uptake and economics.

\section{Materials and Methods}

\section{Experimental site}

Field experiment was conducted at Deendayal Research Institute-Krishi Vigyan Kendra, Chitrakoot UP during rabi season in 2008-09 and 2009-10under irrigated condition in clay loam soils. The experiment site is located in semi-arid and subtropical climate with 850 mm mean annual rainfall. Hot waves during month of March create terminal heat stress condition in the area. The soil is clay loam (sand-21\%, silt-32\% and clay-47\%) having $0.31 \%$ organic carbon with a $\mathrm{pH}$ of 7.34 . The available NPK in the soil were 118.39, 14.96 and $331.50 \mathrm{kgha}^{-}{ }^{1}$, which showed rich in potassium, medium in phosphorus and poor in nitrogen.

\section{Experiment design}

Eighteen treatments comprised residual impact of 3 manures (FYM. Vermi-compost and Cow Pat Pit) with two different doses and 3 doses of inorganic fertilizers used in kharif crop followed by 3 doses of inorganic fertilizers to main crop of wheat in Rabi season. They are: $\mathrm{T}_{1}$ - Control (No fertilizer) No fertilizer, $\mathrm{T}_{2}-50 \%$ NPK(10:40:20) $-100 \%$ NPK, $\mathrm{T}_{3}-75 \%$ NPK (15:60:30) - 75\% NPK, $T_{4}-100 \%$ NPK (20:80:40) - 50\% NPK, $\mathrm{T}_{5}-100 \%$ NPK (20:80:40) - 75\% NPK, $\mathrm{T}_{6}-$ 100\% NPK (20:80:40) - 100\% NPK, $\mathrm{T}_{7}-50 \%$ NPK + FYM 10 t ha $^{-1}-50 \%$ NPK, T $8^{-} 50 \%$ $\mathrm{NPK}+$ VC $5{\text { t } \text { ha- }^{1}-50 \% \text { NPK, }}^{1}-50 \%$ NPK + CPP 3.75kgha- ${ }^{1}-50 \%$ NPK, T $10-50 \%$ NPK + FYM10 t ha- ${ }^{1}-100 \%$ NPK, T $11-50 \%$ NPK + VC 5 t ha- $^{1}-100 \%$ NPK, T $12-50 \%$ NPK + CPP3.75kgha- ${ }^{1}-100 \%$ NPK, $\mathrm{T}_{13}-75 \%$ NPK + FYM 5 t ha- $^{1}-75 \%$ NPK, T $_{14-}-75 \%$ NPK + VC 2.5 t ha- $^{1}-75 \%$ NPK, ${ }_{15}-75 \%$ 
NPK +CPP $1.875 \mathrm{kgha}^{1}{ }^{-}-75 \% \quad \mathrm{NPK}, \quad \mathrm{T}_{16^{-}}$ $100 \%$ NPK + FYM 5 tha $^{-1}-100 \%$ NPK, ${ }^{17}-$ $100 \%$ NPK + VC 2.5 t ha- $^{1}-100 \%$ NPK, T $_{18}-$ $100 \%$ NPK + CPP1.875kgha- ${ }^{1}-100 \%$ NPK and tested in Randomized Block Design with 3 replications.

\section{Crop culture}

The wheat was sown on $29^{\text {th }}$ and $28^{\text {th }}$ Nov.in 2008 and 2009 and harvested on $25^{\text {th }}$ and $26^{\text {th }}$ March in 2009 and 2010, respectively. All necessary production practices viz., weed management, plant protection, irrigation etc. were followed as per standard in whole field during crop growth. Wheat was given 3 irrigations on 18th Dec., $12^{\text {th }}$ Jan. and $9^{\text {th }}$ Feb. during 2008-09and $18^{\text {th }}$ Dec., $10^{\text {th }}$ Jan. and $10^{\text {th }}$ Feb. during 2009-10. No incidence of serious insect or diseases was observed. Crops were harvested manually by sickle from ground level and the total above ground biomass was collected from each plot and seed and straw yield were recorded as per treatments after manually threshing.

\section{Fertilization}

The recommended dose of $100 \%$ NPK to kharif crop and wheat were 20: 80: 40 and 120: 60: $40 \quad \mathrm{~kg} \quad \mathrm{~N}: \mathrm{P}_{2} \quad \mathrm{O}_{5}: \mathrm{K}_{2} \quad \mathrm{Oha}^{-1}$, respectively. In kharif, all amount of NPK were applied as basal in furrows before planting. While, in wheat $1 / 3 \mathrm{~N}+$ full amount of $\mathrm{P}$ and $\mathrm{K}$ were given as basal in furrows before sowing.

The remaining $2 / 3 \mathrm{~N}$ was applied in two equal split doses after first and second irrigation as top dressing. Urea $(46 \% \mathrm{~N})$, DAP $(18 \% \mathrm{~N}$, $\left.46 \% \mathrm{P}_{2} \mathrm{O}_{5}\right)$ and Muriate of Potash $\left(\mathrm{K}_{2} \mathrm{O}\right)$ were used to supply $\mathrm{N}, \mathrm{P}, \mathrm{K}$, respectively. The amount of $\mathrm{N}$ was adjusted with DAP and then urea as per treatments. The FYM (0.43:0.29: $0.73 \%$ N:P: K), vermicompost (1.36:0.23: $0.82 \% \mathrm{~N}: \mathrm{P}: \mathrm{K})$ and cow pat pit (1.68:0.31:
$0.85 \% \mathrm{~N}: \mathrm{P}: \mathrm{K})$ were used as organic manures as per treatments.

\section{Yield attributes}

All-important yield attributing observations like number of spikes plant ${ }^{-1}$, spike length (cm), number of spikelet spike ${ }^{-1}$, number of seeds spike ${ }^{-1}$, seed weight spike $^{-1}(\mathrm{~g})$, seed weight plant $^{-1}(\mathrm{~g})$ and seed test weight $(\mathrm{g})$ were recorded from selected 5 plants of wheat crop after harvesting then averaged to calculate specific data required in particular unit.

\section{Yields}

The plants harvested from net plot were threshed manually and seed taken out. The seed and straw yield of each net plot was recorded in $\mathrm{kg} \mathrm{plot}^{-1}$ and converted in to $\mathrm{tha}^{-1}$.

\section{Nutrient uptake}

The available nitrogen, phosphorus and potassium content in seed and straw were analyzed by modified Kjeldahl method (Bremner and Mulvaney, 1982), Vanadomolybdo-phosphoric yellow colour method (Koenig and Johnson, 1942) and Ammonium acetate method (Jackson, 1971) and their uptake were calculated in $\mathrm{kgha}^{-1}$ according to biological yield of respective plots.

\section{Economics}

Cost of cultivation of each treatment was calculated accordingly inputs used and other cost involved during crop period. Gross return was calculated on market rate of produce and subtracting cost of cultivation, the net return was noted. The B: C ratio was calculated by dividing gross return by cost of cultivation. All monetary parameters were finally converted in to Rs ha ${ }^{-1}$. 
Results and Discussion

Residual effect of INM and direct effect of Inorganic fertilizers on Wheat

\section{Yield attributing parameters}

Application of $100 \%$ NPK through fertilizer to wheat and residual effect of FYM 10 tha $^{-1}+$ $50 \%$ NPK to Kharif crop $\left(\mathrm{T}_{10}\right)$ recorded significantly higher values of all yield attributing characters i.e. number of spikes plant $^{-1}$ (2.3 and 2.4), spike length (10.3 and $10.4 \mathrm{~cm})$, spikelet spike $^{-1}$ (20.7 and 20.6), seeds spike ${ }^{-1}$ (60.20 and 60.33), seed weight spike $^{-1}$ (2.42 and $2.24 \mathrm{~g}$ ), seed weight plant ${ }^{-1}$ (5.37 and $5.38 \mathrm{~g}$ ), and seed test weight (40.53 and $40.54 \mathrm{~g}$ ) during 2008-09 and 2009-10 (Table 1 and 2).

The possible reasons of higher yield attributing parameters may be ascribed due to balanced dose of NPK supply and sufficient accumulation of $\mathrm{N}$ element and organic content through FYM that obviously increases primary vegetative growth and other growth parameters of wheat.

Table.1 Residual impact of INM and direct application of inorganic fertilizers on Yield attributes of Wheat

\begin{tabular}{|c|c|c|c|c|c|c|c|c|}
\hline \multirow{3}{*}{$\begin{array}{c}\text { Treatments } \\
\text { Kharif - Rabi (Wheat) }\end{array}$} & \multicolumn{8}{|c|}{ Yield attributes } \\
\hline & \multicolumn{4}{|c|}{ 2008-09 } & \multicolumn{4}{|c|}{ 2009-10 } \\
\hline & $\begin{array}{l}\text { Spike } \\
\text { length } \\
(\mathrm{cm})\end{array}$ & $\begin{array}{l}\text { No. of } \\
\text { spikes } \\
\mathrm{m}^{-1}\end{array}$ & $\begin{array}{l}\text { No. of } \\
\text { spikes } \\
\text { plant }^{-1}\end{array}$ & $\begin{array}{l}\text { No. of } \\
\text { spikelet } \\
\text { spike }^{-1}\end{array}$ & $\begin{array}{l}\text { Spike } \\
\text { length } \\
(\mathrm{cm})\end{array}$ & $\begin{array}{l}\text { No. of } \\
\text { spikes } \\
\mathrm{m}^{-1}\end{array}$ & $\begin{array}{l}\text { No. of } \\
\text { spikes } \\
\text { plant }^{-1}\end{array}$ & $\begin{array}{l}\text { No. of } \\
\text { spikelet } \\
\text { spike }^{-1}\end{array}$ \\
\hline$T_{1}$ - Control (No fertilizer) - No fertilizer & 5.9 & 29.0 & 1.5 & 12.6 & 5.9 & 27.0 & 1.3 & 13.8 \\
\hline$T_{2}-50 \%$ NPK (10:40:20) - 100\% NPK & 9.9 & 44.3 & 2.2 & 18.3 & 9.9 & 44.6 & 2.2 & 18.6 \\
\hline$T_{3-} 75 \%$ NPK (15:60:30) - 75\% NPK & 9.2 & 39.6 & 1.9 & 16.3 & 9.2 & 39.6 & 1.8 & 16.4 \\
\hline$T_{4}-100 \%$ NPK (20:80:40) - 50\% NPK & 8.3 & 35.0 & 1.7 & 14.4 & 8.4 & 35.3 & 1.7 & 14.3 \\
\hline$T_{5^{-}} 100 \%$ NPK (20:80:40) - 75\% NPK & 9.3 & 40.6 & 1.8 & 16.4 & 9.2 & 40.0 & 1.9 & 16.4 \\
\hline$T_{6}-100 \%$ NPK (20:80:40) - 100\% NPK & 9.9 & 44.6 & 2.2 & 18.4 & 9.9 & 45.0 & 2.2 & 18.6 \\
\hline $\mathrm{T}_{7}-50 \% \mathrm{NPK}+\mathrm{FYM} 10 \mathrm{tha}^{-1}-50 \% \mathrm{NPK}$ & 8.9 & 38.3 & 1.8 & 15.5 & 8.9 & 38.6 & 1.8 & 15.5 \\
\hline $\mathrm{T}_{8^{-}} 50 \% \mathrm{NPK}+\mathrm{VC} 5 \mathrm{t} \mathrm{ha}^{-1}-50 \% \mathrm{NPK}$ & 8.8 & 38.0 & 1.7 & 15.3 & 8.8 & 38.0 & 1.7 & 15.3 \\
\hline $\mathrm{T}_{9}-50 \% \mathrm{NPK}+\mathrm{CPP} 3.75 \mathrm{kgha}^{1}{ }^{-50} \%$ NPK & 8.6 & 35.0 & 1.7 & 14.6 & 8.5 & 35.6 & 1.7 & 14.5 \\
\hline$T_{10}-50 \%$ NPK+FYM10 t ha- ${ }^{1}-100 \%$ NPK & 10.3 & 48.0 & 2.3 & 20.7 & 10.4 & 49.7 & 2.4 & 20.6 \\
\hline $\mathrm{T}_{11}-50 \% \mathrm{NPK}+\mathrm{VC} 5 \mathrm{t} \mathrm{ha}^{1}{ }^{1}-100 \% \mathrm{NPK}$ & 10.0 & 47.3 & 2.2 & 20.2 & 10.3 & 48.0 & 2.3 & 20.1 \\
\hline$T_{12}-50 \%$ NPK+CPP3.75kgha- ${ }^{1}-100 \%$ NPK & 9.8 & 45.3 & 2.1 & 19.0 & 9.9 & 45.3 & 2.2 & 18.8 \\
\hline $\mathrm{T}_{13}-75 \% \mathrm{NPK}+\mathrm{FYM} 5 \mathrm{t} \mathrm{ha}^{1}-75 \%$ NPK & 9.5 & 42.0 & 1.9 & 17.4 & 9.5 & 42.3 & 2.1 & 17.4 \\
\hline $\mathrm{T}_{14}-75 \% \mathrm{NPK}+\mathrm{VC} 2.5 \mathrm{t} \mathrm{ha}^{1}-75 \% \mathrm{NPK}$ & 9.4 & 40.6 & 1.9 & 17.4 & 9.4 & 42.0 & 2.0 & 17.4 \\
\hline $\mathrm{T}_{15}-75 \%$ NPK +CPP1.875 $\mathrm{kgha}^{-1}-75 \%$ NPK & 9.3 & 40.3 & 1.8 & 16.7 & 9.3 & 40.6 & 1.9 & 16.5 \\
\hline$T_{16}-100 \%$ NPK + FYM 5 t ha- ${ }^{1}-100 \%$ NPK & 10.0 & 46.0 & 2.2 & 19.8 & 10.0 & 46.6 & 2.3 & 20.0 \\
\hline $\mathrm{T}_{17}-100 \% \mathrm{NPK}+\mathrm{VC} 2.5 \mathrm{t} \mathrm{ha}^{1}{ }^{1}-100 \%$ NPK & 9.9 & 46.0 & 2.2 & 19.3 & 9.9 & 46.0 & 2.2 & 19.8 \\
\hline $\begin{array}{l}\text { T }_{18}-100 \% \text { NPK+CPP1.875kgha- }{ }^{1}-100 \% \\
\text { NPK }\end{array}$ & 9.8 & 45.3 & 2.2 & 18.6 & 9.9 & 45.3 & 2.2 & 18.7 \\
\hline SEm \pm & 0.19 & 0.95 & 0.11 & 0.22 & 0.04 & 0.25 & 0.06 & 0.18 \\
\hline $\mathrm{CD}(\mathrm{P}=\mathbf{0 . 0 5})$ & 0.56 & 2.75 & 0.31 & 0.63 & 0.09 & 0.73 & 0.17 & 0.50 \\
\hline
\end{tabular}


Table.2 Residual impact of INM and direct application of inorganic fertilizers on Yield attributes of Wheat

\begin{tabular}{|c|c|c|c|c|c|c|}
\hline \multirow[b]{3}{*}{ Treatments } & \multicolumn{6}{|c|}{ Yield attributes } \\
\hline & \multicolumn{3}{|c|}{ 2008-09 } & \multicolumn{3}{|c|}{ 2009-10 } \\
\hline & $\begin{array}{l}\text { No. of } \\
\text { seed } \\
\text { spike }^{-1}\end{array}$ & $\begin{array}{c}\text { Seed } \\
\text { weight g } \\
\text { spike }^{-1}\end{array}$ & $\begin{array}{c}\text { Seed } \\
\text { weight } g \\
1000^{-1}\end{array}$ & $\begin{array}{l}\text { No. of } \\
\text { seed } \\
\text { spike }^{-1}\end{array}$ & $\begin{array}{c}\text { Seed } \\
\text { weight } \mathrm{g} \\
\text { spike }^{-1}\end{array}$ & $\begin{array}{c}\text { Seed } \\
\text { weight } g \\
1000^{-1}\end{array}$ \\
\hline$T_{1}$ - Control (No fertilizer) - No fertilizer & 39.86 & 1.21 & 30.32 & 38.93 & 1.24 & 30.35 \\
\hline $\mathrm{T}_{2}-50 \%$ NPK (10:40:20) - 100\% NPK & 55.0 & 2.16 & 39.26 & 55.06 & 2.18 & 39.29 \\
\hline$T_{3^{-}} 75 \%$ NPK (15:60:30) - 75\% NPK & 48.73 & 1.81 & 37.13 & 48.60 & 1.78 & 37.43 \\
\hline$T_{4}-100 \%$ NPK (20:80:40) - 50\% NPK & 42.20 & 1.51 & 35.76 & 41.26 & 1.45 & 35.98 \\
\hline$T_{5^{-}} 100 \%$ NPK (20:80:40) - 75\% NPK & 48.93 & 1.82 & 37.19 & 48.46 & 1.78 & 37.41 \\
\hline $\mathrm{T}_{6}-100 \%$ NPK (20:80:40) - 100\% NPK & 55.33 & 2.17 & 39.21 & 55.13 & 2.18 & 39.30 \\
\hline $\mathrm{T}_{7}-50 \% \mathrm{NPK}+\mathrm{FYM} 10 \mathrm{tha}^{-1}-50 \% \mathrm{NPK}$ & 47.00 & 1.87 & 39.81 & 47.06 & 1.88 & 39.82 \\
\hline $\mathrm{T}_{8^{-}} 50 \% \mathrm{NPK}+\mathrm{VC} 5 \mathrm{t} \mathrm{ha}^{-}{ }^{-}-50 \% \mathrm{NPK}$ & 46.00 & 1.86 & 39.76 & 46.93 & 1.85 & 39.76 \\
\hline $\mathrm{T}_{9}-50 \% \mathrm{NPK}+\mathrm{CPP} 3.75 \mathrm{kgha}^{-}{ }^{-}-50 \% \mathrm{NPK}$ & 42.73 & 1.56 & 36.50 & 41.40 & 1.56 & 36.51 \\
\hline $\mathrm{T}_{10}-50 \% \mathrm{NPK}+\mathrm{FYM}_{10} 10 \mathrm{t} \mathrm{ha}^{-1}-100 \% \mathrm{NPK}$ & 60.20 & 2.42 & 40.53 & 60.33 & 2.24 & 40.54 \\
\hline $\mathrm{T}_{11}-50 \% \mathrm{NPK}+\mathrm{VC} 5 \mathrm{t} \mathrm{ha-}^{1}-100 \%$ NPK & 59.73 & 2.40 & 40.51 & 59.86 & 2.23 & 40.51 \\
\hline $\mathrm{T}_{12}-50 \% \mathrm{NPK}+\mathrm{CPP} 3.75 \mathrm{kgha}^{1}-100 \%$ NPK & 55.80 & 2.22 & 39.75 & 55.60 & 2.19 & 39.75 \\
\hline$T_{13}-75 \%$ NPK + FYM 5 t ha- ${ }^{1}-75 \%$ NPK & 51.60 & 2.00 & 38.78 & 52.33 & 2.05 & 38.78 \\
\hline$T_{14}-75 \%$ NPK + VC 2.5 t ha- ${ }^{1}-75 \%$ NPK & 51.26 & 2.00 & 39.00 & 51.93 & 2.02 & 38.77 \\
\hline $\mathrm{T}_{15}-75 \% \mathrm{NPK}+\mathrm{CPP} 1.875 \mathrm{kgha}^{-}-75 \%$ NPK & 47.26 & 1.80 & 38.08 & 48.86 & 1.82 & 38.08 \\
\hline$T_{16}-100 \%$ NPK + FYM 5 t ha- ${ }^{1}-100 \%$ NPK & 55.73 & 2.23 & 40.00 & 55.86 & 2.19 & 40.04 \\
\hline $\mathrm{T}_{17}-100 \% \mathrm{NPK}+\mathrm{VC} 2.5 \mathrm{t}$ ha- ${ }^{1}-100 \% \mathrm{NPK}$ & 55.33 & 2.20 & 39.76 & 55.53 & 2.19 & 39.93 \\
\hline $\mathrm{T}_{18}-100 \% \mathrm{NPK}+\mathrm{CPP} 1.875 \mathrm{kgha}^{1}{ }^{-}-100 \%$ NPK & 54.80 & 2.18 & 39.75 & 55.40 & 2.16 & 39.80 \\
\hline SEm \pm & 0.64 & 0.06 & 2.72 & 0.33 & 0.04 & 0.16 \\
\hline
\end{tabular}

Table.3 Residual impact of INM and direct application of inorganic fertilizers on Yields of Wheat

\begin{tabular}{|c|c|c|c|c|c|c|}
\hline \multirow{3}{*}{$\begin{array}{c}\text { Treatment } \\
\text { Kharif - Rabi (Wheat) }\end{array}$} & \multicolumn{4}{|c|}{ Yield } & \multirow{2}{*}{\multicolumn{2}{|c|}{ Harvest Index (\%) }} \\
\hline & \multicolumn{2}{|c|}{ Seed $\left(\mathbf{t ~ h a} \mathbf{a}^{-1}\right)$} & \multicolumn{2}{|c|}{ Straw $\left(\mathbf{t ~ h a}^{-1}\right)$} & & \\
\hline & 2008-09 & 2009-10 & 2008-09 & 2009-10 & 2008-09 & 2009-10 \\
\hline$T_{1}$ - Control (No fertilizer) - No fertilizer & 1.55 & 1.49 & 1.56 & 1.57 & 49.25 & 48.72 \\
\hline $\mathrm{T}_{2^{-}} 50 \%$ NPK $(10: 40: 20)-100 \%$ NPK & 4.29 & 4.32 & 4.40 & 4.47 & 49.37 & 49.14 \\
\hline $\mathrm{T}_{3^{-}} 75 \% \mathrm{NPK}(15: 60: 30)-75 \% \mathrm{NPK}$ & 3.09 & 3.13 & 3.23 & 3.36 & 48.69 & 48.19 \\
\hline $\mathrm{T}_{4^{-}} 100 \% \mathrm{NPK}(20: 80: 40)-50 \% \mathrm{NPK}$ & 2.23 & 2.248 & 2.32 & 2.34 & 49.80 & 48.94 \\
\hline $\mathrm{T}_{5^{-}} 100 \%$ NPK $(20: 80: 40)-75 \%$ NPK & 3.11 & 3.17 & 3.26 & 3.34 & 48.84 & 48.72 \\
\hline $\mathrm{T}_{6}-100 \% \mathrm{NPK}(20: 80: 40)-100 \% \mathrm{NPK}$ & 4.31 & 4.34 & 4.49 & 4.52 & 48.93 & 49.02 \\
\hline $\mathrm{T}_{7^{-}} 50 \% \mathrm{NPK}+\mathrm{FYM} 10 \mathrm{tha}^{-1}-50 \% \mathrm{NPK}$ & 3.12 & 3.22 & 3.48 & 3.51 & 47.26 & 48.34 \\
\hline $\mathrm{T}_{8^{-}} 50 \% \mathrm{NPK}+\mathrm{VC} 5$ t ha-1 - 50\% NPK & 3.08 & 3.11 & 3.39 & 3.42 & 47.57 & 47.66 \\
\hline $\mathrm{T}_{9^{-}} 50 \% \mathrm{NPK}+\mathrm{CPP} 3.75 \mathrm{kgha}-1-50 \% \mathrm{NPK}$ & 2.42 & 2.48 & 2.55 & 2.66 & 48.66 & 48.41 \\
\hline $\mathrm{T}_{10}-50 \% \mathrm{NPK}+\mathrm{FYM} 10 \mathrm{t}$ ha-1 $-100 \% \mathrm{NPK}$ & 4.94 & 4.95 & 4.95 & 5.04 & 49.95 & 49.54 \\
\hline $\mathrm{T}_{11}-50 \% \mathrm{NPK}+\mathrm{VC} 5 \mathrm{t}$ ha-1 $-100 \% \mathrm{NPK}$ & 4.88 & 4.76 & 4.93 & 4.93 & 49.73 & 49.14 \\
\hline $\mathrm{T}_{12}-50 \% \mathrm{NPK}+\mathrm{CPP} 3.75 \mathrm{kgha}-1-100 \% \mathrm{NPK}$ & 4.38 & 4.41 & 4.57 & 4.58 & 49.04 & 49.02 \\
\hline $\mathrm{T}_{13}-75 \%$ NPK + FYM 5 t ha-1 - 75\% NPK & 4.18 & 3.86 & 4.59 & 4.21 & 47.65 & 47.87 \\
\hline $\mathrm{T}_{14}-75 \% \mathrm{NPK}+\mathrm{VC} 2.5 \mathrm{t}$ ha- $1-75 \% \mathrm{NPK}$ & 4.09 & 3.82 & 4.52 & 4.16 & 47.50 & 47.86 \\
\hline $\mathrm{T}_{15}-75 \% \mathrm{NPK}+\mathrm{CPP} 1.875$ kgha-1 - 75\% NPK & 3.26 & 3.24 & 3.60 & 3.59 & 47.51 & 47.40 \\
\hline $\mathrm{T}_{16}-100 \% \mathrm{NPK}+\mathrm{FYM} 5 \mathrm{t}$ ha-1 - 100\% NPK & 4.51 & 4.53 & 4.55 & 4.68 & 49.73 & 49.17 \\
\hline $\mathrm{T}_{17}-100 \% \mathrm{NPK}+\mathrm{VC} 2.5 \mathrm{t}$ ha- $-1-100 \% \mathrm{NPK}$ & 4.41 & 4.46 & 4.48 & 4.63 & 49.64 & 49.05 \\
\hline $\mathrm{T}_{18}-100 \% \mathrm{NPK}+\mathrm{CPP} 1.875 \mathrm{kgha}-1-100 \% \mathrm{NPK}$ & 4.36 & 4.36 & 4.45 & 4.58 & 49.46 & 48.78 \\
\hline $\operatorname{SEm} \pm$ & 0.25 & 0.35 & 0.22 & 0.21 & 0.39 & 0.25 \\
\hline $\mathrm{CD}(\mathrm{P}=0.05)$ & 0.73 & 0.72 & 0.63 & 0.59 & 1.12 & 0.74 \\
\hline
\end{tabular}


Table.4 Residual impact of INM and direct application of inorganic fertilizers on Nutrient uptake of Wheat

\begin{tabular}{|c|c|c|c|c|c|c|}
\hline \multirow{3}{*}{$\begin{array}{c}\text { Treatment } \\
\text { Kharif - Wheat }\end{array}$} & \multicolumn{6}{|c|}{ Nutrient uptake (kgha-1) } \\
\hline & \multicolumn{2}{|c|}{ Nitrogen } & \multicolumn{2}{|c|}{ Phosphorus } & \multicolumn{2}{|c|}{ Potassium } \\
\hline & 2008-09 & 2009- 10 & 2008-09 & 2009- 10 & 2008-09 & 2009- 10 \\
\hline $\mathrm{T}_{1}$ - Control (No fertilizer) - No fertilizer & 32.6 & 31.3 & 4.6 & 4.3 & 13.6 & 13.0 \\
\hline $\mathrm{T}_{2^{-}} 50 \%$ NPK (10:40:20)- 100\% NPK & 108.0 & 107.6 & 15.3 & 15.6 & 64.3 & 66.0 \\
\hline $\mathrm{T}_{3}-75 \%$ NPK (15:60:30) - 75\% NPK & 67.0 & 67.6 & 12.0 & 12.0 & 42.6 & 44.3 \\
\hline $\mathrm{T}_{4}-100 \%$ NPK $(20: 80: 40)-50 \%$ NPK & 55.6 & 57.0 & 7.3 & 8.0 & 27.6 & 27.3 \\
\hline $\mathrm{T}_{5^{-}} 100 \%$ NPK (20:80:40) - 75\% NPK & 70.3 & 71.6 & 12.3 & 12.6 & 45.3 & 45.0 \\
\hline $\mathrm{T}_{6}-100 \%$ NPK $(20: 80: 40)-100 \%$ NPK & 107.6 & 111.6 & 16.3 & 16.3 & 68.6 & 69.3 \\
\hline $\mathrm{T}_{7}-50 \% \mathrm{NPK}+\mathrm{FYM} 10 \mathrm{t}$ ha-1 $-50 \% \mathrm{NPK}$ & 81.3 & 82.6 & 11.0 & 11.6 & 49.6 & 51.3 \\
\hline $\mathrm{T}_{8^{-}}-50 \% \mathrm{NPK}+\mathrm{VC} 5 \mathrm{t}$ ha-1 $-50 \% \mathrm{NPK}$ & 77.0 & 77.3 & 11.0 & 11.0 & 44.6 & 48.3 \\
\hline $\mathrm{T}_{9^{-}} 50 \% \mathrm{NPK}+\mathrm{CPP} 3.75 \mathrm{kgha}-1-50 \% \mathrm{NPK}$ & 60.3 & 61.6 & 10.0 & 9.6 & 31.0 & 33.3 \\
\hline $\mathrm{T}_{10}-50 \% \mathrm{NPK}+\mathrm{FYM} 10 \mathrm{t}$ ha- $1-100 \%$ NPK & 119.0 & 123.3 & 20.3 & 20.0 & 82.0 & 83.6 \\
\hline $\mathrm{T}_{11}-50 \% \mathrm{NPK}+\mathrm{VC} 5 \mathrm{t}$ ha- $1-100 \% \mathrm{NPK}$ & 114.3 & 116.0 & 20.0 & 19.6 & 78.6 & 80.6 \\
\hline $\mathrm{T}_{12}-50 \% \mathrm{NPK}+\mathrm{CPP} 3.75$ kgha-1 $-100 \% \mathrm{NPK}$ & 103.3 & 106.0 & 16.0 & 17.0 & 69.3 & 69.6 \\
\hline $\mathrm{T}_{13}-75 \% \mathrm{NPK}+\mathrm{FYM} 5 \mathrm{t}$ ha-1 $-75 \% \mathrm{NPK}$ & 94.6 & 95.0 & 16.0 & 16.0 & 63.0 & 65.6 \\
\hline $\mathrm{T}_{14}-75 \% \mathrm{NPK}+\mathrm{VC} 2.5 \mathrm{t}$ ha- $1-75 \% \mathrm{NPK}$ & 94.0 & 94.6 & 15.3 & 15.6 & 60.0 & 63.0 \\
\hline $\mathrm{T}_{15}-75 \% \mathrm{NPK}+\mathrm{CPP} 1.875$ kgha-1- $75 \% \mathrm{NPK}$ & 71.3 & 72.0 & 13.0 & 13.0 & 47.6 & 50.0 \\
\hline $\mathrm{T}_{16}-100 \%$ NPK + FYM 5 t ha-1 - 100\% NPK & 114.3 & 114.6 & 19.0 & 19.3 & 71.6 & 78.0 \\
\hline $\mathrm{T}_{17}-100 \% \mathrm{NPK}+\mathrm{VC} 2.5 \mathrm{t}$ ha-1 $-100 \% \mathrm{NPK}$ & 109.3 & 110.0 & 18.0 & 18.0 & 68.6 & 75.3 \\
\hline $\mathrm{T}_{18}-100 \% \mathrm{NPK}+\mathrm{CPP} 1.875 \mathrm{kgha}-1-100 \% \mathrm{NPK}$ & 106.3 & 108.3 & 16.3 & 16.0 & 66.3 & 71.6 \\
\hline SEm \pm & 0.55 & 0.62 & 0.33 & 0.42 & 0.56 & 0.67 \\
\hline $\mathrm{CD}(\mathrm{P}=0.05)$ & 1.58 & 1.78 & 0.94 & 1.22 & 1.63 & 1.93 \\
\hline
\end{tabular}

Table.5 Residual impact of INM and direct application of inorganic fertilizers on economics of Wheat

\begin{tabular}{|c|c|c|c|c|c|c|c|c|}
\hline \multirow{3}{*}{ Treatments } & \multicolumn{8}{|c|}{ Economics } \\
\hline & \multicolumn{2}{|c|}{$\begin{array}{l}\text { Cost of cultivation } \\
\left(\mathrm{Rs} \mathrm{ha}^{-1}\right)\end{array}$} & \multicolumn{2}{|c|}{$\begin{array}{l}\text { Gross Return } \\
\quad\left(\mathrm{Rs} \mathrm{ha}^{-1}\right)\end{array}$} & \multicolumn{2}{|c|}{$\begin{array}{l}\text { Net Return } \\
\left(\mathrm{Rs} \mathrm{ha}^{-1}\right)\end{array}$} & \multicolumn{2}{|c|}{ B:C Ratio } \\
\hline & 2008-09 & 2009-10 & 2008-09 & 2009-10 & 2008-09 & 2009-10 & 2008-09 & 2009-10 \\
\hline$T_{1}$ - Control (No fertilizer) - No fertilizer & 9214 & 11195 & 17061 & 18827 & 7847 & 7632 & 1.85 & 1.68 \\
\hline$T_{2}-50 \%$ NPK (10:40:20) - 100\% NPK & 11893 & 13870 & 47428 & 54417 & 35535 & 40547 & 3.99 & 3.92 \\
\hline$T_{3-}-75 \%$ NPK (15:60:30) - 75\% NPK & 11205 & 13186 & 34360 & 39720 & 23155 & 26534 & 3.06 & 3.01 \\
\hline$T_{4}-100 \%$ NPK $(20: 80: 40)-50 \%$ NPK & 10520 & 12507 & 24721 & 28225 & 14201 & 15718 & 2.35 & 2.25 \\
\hline$T_{5^{-}} 100 \%$ NPK (20:80:40) - 75\% NPK & 11205 & 12852 & 34506 & 40027 & 23301 & 27175 & 3.08 & 3.04 \\
\hline$T_{6}-100 \%$ NPK (20:80:40) - 100\% NPK & 11993 & 13870 & 47744 & 54721 & 35851 & 40851 & 4.01 & 3.94 \\
\hline $\mathrm{T}_{7}-50 \% \mathrm{NPK}+\mathrm{FYM} 10 \mathrm{t} \mathrm{ha}^{-1}-50 \% \mathrm{NPK}$ & 10520 & 12507 & 35055 & 41004 & 24535 & 28497 & 3.33 & 3.28 \\
\hline $\mathrm{T}_{8^{-}}-50 \% \mathrm{NPK}+\mathrm{VC} 5 \mathrm{t} \mathrm{ha-}^{1}-50 \% \mathrm{NPK}$ & 10520 & 12507 & 34491 & 39668 & 23971 & 27161 & 3.28 & 3.17 \\
\hline $\mathrm{T}_{9-}-50 \% \mathrm{NPK}+\mathrm{CPP} 3.75 \mathrm{kgha}^{1}{ }^{-}-50 \%$ NPK & 10520 & 12507 & 26899 & 31631 & 16379 & 19124 & 2.56 & 2.53 \\
\hline $\mathrm{T}_{10}-50 \% \mathrm{NPK}+\mathrm{FYM} 10 \mathrm{t} \mathrm{ha}^{-1}-100 \% \mathrm{NPK}$ & 11893 & 13870 & 54372 & 62083 & 42479 & 48213 & 4.57 & 4.48 \\
\hline$T_{11}-50 \% \mathrm{NPK}+$ VC $5 \mathrm{t}^{\text {ha- }}{ }^{1}-100 \% \mathrm{NPK}$ & 11893 & 13870 & 53756 & 59921 & 41863 & 46.51 & 4.52 & 4.30 \\
\hline $\mathrm{T}_{12}-50 \% \mathrm{NPK}+\mathrm{CPP} 3.75 \mathrm{kgha}^{-1}-100 \%$ NPK & 11893 & 13870 & 48559 & 55525 & 36666 & 41655 & 4.08 & 4.00 \\
\hline $\mathrm{T}_{13}-75 \% \mathrm{NPK}+$ FYM $5 \mathrm{t} \mathrm{ha-}^{1}-75 \%$ NPK & 11205 & 13186 & 46794 & 49171 & 35589 & 35985 & 4.18 & 3.73 \\
\hline$T_{14}-75 \%$ NPK + VC 2.5 t ha- $^{1}-75 \%$ NPK & 11205 & 13186 & 45899 & 48604 & 34694 & 35418 & 4.10 & 3.69 \\
\hline $\mathrm{T}_{15}-75 \% \mathrm{NPK}+\mathrm{CPP} 1.875 \mathrm{kgha}^{-}-75 \% \mathrm{NPK}$ & 11205 & 13186 & 36531 & 41383 & 25326 & 28197 & 3.26 & 3.14 \\
\hline $\mathrm{T}_{16}-100 \% \mathrm{NPK}+\mathrm{FYM} 5 \mathrm{t} \mathrm{ha-}^{1}-100 \%$ NPK & 11893 & 13870 & 49673 & 57025 & 37780 & 43155 & 4.18 & 4.11 \\
\hline $\mathrm{T}_{17}-100 \% \mathrm{NPK}+\mathrm{VC} 2.5 \mathrm{t} \mathrm{ha-}^{1}-100 \% \mathrm{NPK}$ & 11893 & 13870 & 48658 & 56141 & 36765 & 42271 & 4.09 & 4.05 \\
\hline$T_{18}-100 \% N P K+C P P 1.875$ kgha- $^{1}-100 \%$ NPK & 11893 & 13870 & 48131 & 55062 & 36238 & 41192 & 4.05 & 3.97 \\
\hline SEm \pm & - & - & 511.0 & 3518.0 & 533.4 & 834.4 & 0.03 & 0.06 \\
\hline $\mathrm{CD}(\mathrm{P}=\mathbf{0 . 0 5})$ & - & - & 1469 & 10117 & 1533 & 2410 & 0.13 & 0.18 \\
\hline
\end{tabular}


Balanced application of fertilizers and credited macro and micronutrients through organic manures, used in preceding kharif crop enhanced the root and shoot development. Since adequate supply of $\mathrm{N}$ promotes the physiological process of growth and development of wheat crop and ultimately gave higher values of yield attributing characters (Table 1 and 2). These results have also reported bySingh et al., (2011), Thakur et al., (2011), Meena et al., (2013), Usadadiya et al., (2013), Vinay Singh (2016) and Parewa et al., (2019).

\section{Yield and Harvest Index}

Application of $100 \%$ NPK to wheat and residual effect of FYM @ 10 tha $^{-1}+50 \%$ NPK to previous kharif crop $\left(\mathrm{T}_{10}\right)$ recorded significantly higher seed (4.94 and 4.95 $\mathrm{t} \mathrm{ha-}^{1}$ ), straw yield (4.95 and $5.04 \mathrm{t} \mathrm{ha}^{1}$ ) and Harvest index (49.95 and $49.54 \%$ ) of wheat over rest of the treatments except $100 \%$ NPK with residual impact of vermicompost @5 tha $^{-1}$ and $50 \%$ NPK $\left(\mathrm{T}_{11}\right)$ which was statistically at par with $\mathrm{T}_{10}$ during 2008-09 and 2009-10 (Table 3). This was obtained due to accumulated $\mathrm{C}: \mathrm{N}$ ratio at primary stage of the crop (Pratap et al., 2006). Nitrogen directly influenced plant growth and development of crop canopy through better utilization of photosynthates. Moreover, at maturity stage of crop more photosynthates are diverted and accumulated in spike resulted to maximum grain yield. Further optimum dose of phosphorus and potassium played their role for development of vigorous seed and a greater number of seeds in spike. This was because the $\mathrm{N}$ omission strongly depressed the grain and straw production of wheat (Hussain and Kumar, 2013). The residual $\mathrm{N}$ also helped to sustain yield improvement in wheat and sustained the productivity Aulakh et al., (2012). Residual impact of manures in wheat crop exerted significant effect on grain and straw yield over $100 \%$ NPK alone (Pandey and Kumar 2017). Residual macro and microelement through FYM provided better soil environment and physical condition for better root development, which able to supply addition demand of mineral and water for maximum photosynthesis process. These findings are similar those of Usadadiya et al., (2013), Das et al., (2015), Singh et al., (2015), Nawale et al., (2018) and Parewa et al., (2019).

\section{Nutrient uptake}

Nitrogen (119.0 and $\left.123.3 \mathrm{~kg} \mathrm{ha}^{-1}\right)$ and potassium (82.0 and $\left.83.6 \mathrm{~kg} \mathrm{ha}^{-1}\right)$ uptake of wheat were found significantly higher with residual impact of 100\% NPK + FYM @ 10 tha ${ }^{-1}$ and $100 \%$ NPK to wheat $\left(\mathrm{T}_{10}\right)$ over other treatments during 2008-09 and 2009-10. While, phosphorus uptake (20.3 and $20.0 \mathrm{~kg}$ $\mathrm{ha}^{-1}$ ) was recorded significantly greater under same treatment but it was statistically at par with $\mathrm{T}_{11}$ (residual effect of $100 \% \mathrm{NPK}+$ vermicompost @ 5 tha $^{-1}$ and $100 \%$ NPK to wheat) in both the years (Table 4). It might be due to use of balanced dose of NPK to wheat with residual response of organic manures and inorganic N \& P. Consequently, higher soil microbial activity with the presence of organic matter, which ensured higher availability of NPK thus it, increased root cation exchange capacity. Jointly these owing to increase supply of nutrient to the crop, as well as reduced the loss of organically supplied nutrients. Das et al., (2015), Sharma et.al. (2015), Singh et al., (2015), Vinay Singh (2016), Dwivedi et al., (2016), Singh and Patra (2017), Nawaleet.al., (2018), Bairwa et al., (2019) and Paramesh et al., (2020) also reported similar findings.

\section{Economics}

Cost of cultivation of wheat was recorded maximum Rs. 11893 and $13870 \mathrm{ha}^{-1}$ during 
2008-09 and 2009-10 in those treatment having $100 \%$ RDF with inorganic fertilization followed by $75 \%$ and $50 \%$ NPK of RDF. The gross return (Rs 54372 and $62.083 \mathrm{ha}^{-1}$ ), net return (Rs. 42479 and $48213 \mathrm{ha}^{-1}$ ) and B:C ratio (4.57 and 4.48) were recorded significantly higher under treatment $\mathrm{T}_{10}$ (residual effect of 100\% NPK+FYM @ 5 $\mathrm{t} \mathrm{ha}^{-1}$ in kharif crop) in compare to all other treatments during 2008-09 and 2009-10 (Table 5).

The economic output of wheat influenced significantly with doses of Fertilizers (50, 75 and $100 \%$ ) and residual effect of inorganic fertilizers and Manures (FYM, Vermicompost and CPP) which were used in preceding crop. Certainly, the higher values of economic return were directly related to higher values of grain and straw yield and cost of cultivation are higher side due to higher cost of vermi-compost and FYM in compare to other treatment. Earlier findings in this regard reported by Hussain et al., (2013) Das et al., (2015, Sharma et al., (2015), Vinay Singh (2016), Singh and Singh (2018), Bairwa et al., (2019) and Kumar et al., (2020) had also showed conformity.

In conclusion the study provides information that residual effect of FYM 5 tha ${ }^{-1}$ with $100 \%$ recommended dose of inorganic fertilizer to previous crop and $100 \%$ RDF to wheat was found as a best combination of organic manure and inorganic fertilizers in term of yield attributes, yield, nutrient uptake and economics of wheat.

\section{Acknowledgement}

I acknowledge my gratitude toward Deendayal Research institute to facilitate to conduct this trial and also to Indian Council of Agriculture Research to facilitate required budget of this experiment during both the years.

\section{References}

Aulakh M S, Jogider S, Manchanda, Garg AG, Kumar S, DerconG and MinhLong N (2012). Crop production and nutrient use efficiency of conservation agriculture for soybean - whaet rotation on the Indo-Gangatic plains of Northwestern India. Soil and Tillage Research. 120: 50-60.

Bairwa P C, SammauriaR andGupta K C (2019). Direct and Residual Effect of PROM on Productivity, Nutrient Uptake, Soil Properties and Economics under Clusterbean-Wheat Cropping System. Journal of Soil Salinity and Water Quality, 11(1), 84-89.

Bremner JM and Malvaney CS (1982). Nitrogen total. In: Methods of Soil Analysis, part 2, chemical and microbiological properties Agronomy monograph no. 9: 595-624. Am. Soc. Agron. Inc. Madison Wisconsin, USA.

Chandel, B.S., Singh, Sandeep, Singh, H. and Singh, V. (2014) Direct and residual effect of nutrient management in wheatmaize cropping sequence. Journal of the Indian Society Soil Science, 62(2): 126- 130.

Das D, Dwivedi B S, Meena M C, Singh V K and Tiwari K N (2015). Integrated nutrient management for improving soil health and crop productivity. Indian journal of Fertilizer, 11(4) 64-83.

Dhakal Y, Meena R S and Kumar S (2016). Effect of INM on nodulation, yield, quality and available nutrient balance in soil after harvest of green gram. Legume Res. 39(4):590-594.

Dwivedi B S, Singh V K, Meena M C, Dey A and Dutta S P (2016). Integrated nutrient management for enhancing nutrient use efficiency. Indian journal of Fertilizer. 12(44):62-71.

Hussain, A. and Kumar, D (2013). Productivity, nutrient concentration and 
uptake of wheat as affected by nutrient omissions in cotton-wheat cropping system. Indian Journal of Fertilizers, 9(3): 34-41.

Hussain, A. and Kumar, D., Rana, D.S., Gangaiah, B., Dwivedi, B.S. and Sahoo, R.N. (2013). Productivity and profitability of $\mathrm{Bt}$ cotton (Gossypium hirsutum) wheat (Triticum aestivum) cropping system as affected by nutrient omission. Indian Journal of Agronomy, 58(2): 175-181.

JacksonM L (1971). Soil chemical analysis, Prentice Hall of India Pvt. Ltd. New Delhi pp. 498.

Jat L K, Singh Y V, Meena S K, Meena S Kumari, Parihar M, Jatav M S, Meena R K and Meena V S (2015). Does Integrated nutrient management enhance agricultural productivity. Journal of pure and applied microbiology. 9(2)1211-1221.

KoenigRA and Johnson CR (1942). Colorimetric determination of $\mathrm{P}$ in biological materials. Ind. Eng. Chem. Anal. 14: 155-56.

Kumar, M, Bauddh, K, Kumar, S, Sainger, M,Sainger. P A and Singh, RP (2013). Increase in growth, productivity and nutritional status of wheat (Triticum aestivum L. cv. WH-711) and enrichment in soil fertility applied with organic matrix entrapped urea. $J$. Environmental Biology. 34: 1-9.

Maneesh K, Singh S K and Bohra J S (2020). Cumulative Effect of Organic and Inorganic Sources of Nutrients on Yield, Nutrients Uptake and Economics by Rice-Wheat Cropping System in Indo-Gangetic Plains of India. Communications in Soil Science and Plant Analysis51 (5): 658-674.

Meena V S, Maurya B R, Verma R, Meena R, Meena R S, Jatav G K and Singh D K (2013).Influence of growth and yield attributes of wheat (Triticum aestivum
L.) By organic and inorganic sources of nutrients with residual effect under different fertility levels. The Bioscan. 8(3):811-815

Nawale, S S, Kolekar P T and Solanke AU (2018). Soybean followed by wheat cropping system under variable sowing windows and fertilizer levels. Journal of Pharmacology and Phytochemistry. 7(1):2181-2185.

Pandey, M. and Kumar, M (2017). Effect of sulphur, manganese and zinc on yield, quality and uptake of nutrients by wheat (Triticum aestivum). Annals of Plant and Soil Research, 19(4): 403407

Parewa H P, Ram M, Jain L K, Chaudhary A and Ratnoo S D (2019). Impact of Organic Nutrient Management Practices on Yield Attributes, Yield and Economics of Wheat (Triticum aestivum L.). International Journal of Bio-resource and Stress Management. 10(3): 257- 260.

Paramesh, V, Dhar, S,Dass, A, Kumar, B, Kumar, A, El-Ansary, D.O andElansary, HO (2020). Role of Integrated Nutrient Management and Agronomic Fortification of Zinc on Yield, Nutrient Uptake and Quality of Wheat. Sustainability 12: 3513.

Pratap-Singh, Nepalia, V. and Tomar, S.S (2006). Effect of weed control and nutrient management on soybean (Glycine max) productivity. Indian Journal of Agronomy. 51(4): 314-317.

Sharma G.D., Thakur Risikesh, Chouhan Nar endra and Keram K.S (2015). Effect of Integrated Nutrient Management on Yield, Nutrient Uptake, Protein Content, Soil Fertility and Economic Performance of Rice (Oryza sativa L.) in a Vertisol. Journal of the Indian Society of Soil Science.63 (3): 320-326.

Singh, G., Singh, O. P., Singh, S. and Prasad $\mathrm{K}$ (2010). Weed management in late 
sown wheat (Triticum aestivum) after rice (Oryza sativa) in rice-wheat system in rainfed lowland. Indian Journal of Agronomy 55(2): 83-88.

Singh, CM, Sharma, P K, Kishor P, Mishra, P K, Singh, AP, Verma, R., Raha, P (2011). Impact of integrated nutrient management on growth, yield and nutrient uptake by wheat (Triticum aestivum L.). Asian Journal Agricultural Research. 5(1):76-82.

Singh V, Ali J, Seema, Kumar A, Chauhan T M (2015). Productivity, nutrient uptake and economics of wheat (Triticum aestivum) under potassium and zinc nutrition. Indian Journal of Agronomy. 60 (3): 426- 430.

Singh, V. and Patra, A (2017). Effect of FYM and manganese on yield and uptake of nutrients in wheat (Triticum aestivum). Annals of Plant and Soil Research, 19 (4): 381-384.

Singh, S. and Singh, V (2018) Maximizing wheat (Triticum aestivum) productivity and profitability using site specific nutrient management strategy. Annals of Plant and Soil Research, 20(1): 103106.

Thakur R, Sawarkar S D, Vaishya U K, Singh M (2011). Impact of continuous use of inorganic fertilizers and organic manure on soil properties and productivity under soybean - wheat intensive cropping of a vertisol. Journal of the Indian Society of Soil Science. 59 (1):74-81.

Usadadiya VP and Patel RH (2013). Influence of preceding crops and nutrient management on productivity of wheat (Triticum aestivum)-based cropping system. Indian Journal of Agronomy. 58(1):15-18

Vinay Singh (2016). Productivity, nutrient uptake and economics of wheat as affected by nutrient omissions in alluvial soil. Annals of Plant and Soil Research, 18(3): 219-225.

\section{How to cite this article:}

Narendra Singh and Kushwaha, H. S. 2020. Residual Impact of Integrated Nutrient Management on Yield Attributes, Yields, Nutrient Uptake and Economics in Wheat (Triticum aestivum). Int.J.Curr.Microbiol.App.Sci. 9(09): 1146-1155.

doi: https://doi.org/10.20546/ijcmas.2020.909.142 